\title{
1995 EPS High Energy \& Particle Physics Prize
}

The EPS High Energy and Particle Physics Division (HEPP) has awarded the 1995 HighEnergy and Particle Physics Prize to:

Paul Söding, DESY-Institute of High-Energy Physics, Zeuthen

Björn Wiik, Deutsches Elektronen-Synchrotron (DESY), Hamburg

Günther Wolf, DESY, Hamburg

Sau Lan Wu, University of Wisconsin, Madison, Wisconsin, USA.

for the first evidence for three-jet events in $\mathrm{e}^{+} \mathrm{e}^{-}$collisions at PETRA, the $2.3 \mathrm{~km}$ in circumference storage ring at DESY which started operating in November 1978.

This important experimental contribution, first reported in June 1979, was the result of a large joint effort by accelerator and experimental particle physicists based both at DESY and collaborating institutes. It was obtained while the prizewinners were working with the TASSO detector, one of the four experiments initially installed at PETRA. It generated considerable interest and stimulated an enormous analytical effort because a possible interpretation invoked the creation of gluons by quark bremsstrahlung (in three-jet events, two jets - small bundles of several high-energy particles - originate from a quark and an anti-quark and a third jet from a radiated gluon).

The Prize was presented by the Chairman of the HEPP Division to the four prizewinners at the 1995 International Europhysics Conference on High Energy Physics (Brussels; 27 July 2 August 1995)

\section{Special EPS High Energy \& Particle Physics Prize}

The Executive Committee of EPS, in agreement with the Chairman of the EPS High Energy and Particle Physics (HEPP) Division, has awarded a special complementary Prize to:

The JADE Collaboration, Deutsches Elektronen-Synchrotron (DESY), Hamburg The MARK J Collaboration, DESY

The PLUTO Collaboration, DESY

The TASSO Collaboration, DESY,

each responsible for one of the four detectors initially installed at DESY's PETRA storage ring.

While the 1995 HEPP Prize is in recognition of the observation of three-jet events representing a first indication of the existence of the gluon, confirmation of the existence of this important fundamental particle emerged gradually in several independent ways from data obtained by the four Collaborations, all of which presented results for gluon bremsstrahlung in August 1979 at the Lepton-Photon Conference (Batavia, IL, USA). Confirming the existence of the gluon, the carrier particle for the strong interaction, was of fundamental importance for the Standard Model of particle physics. In view of this, and because the HEPP Prize can only be awarded to nominated individuals, the special Prize supplements the 1995 HEPP Prize by recognizing the Collaborations' achievements.

The Prize was announced by the Chairman of the HEPP Division at the 1995 International Europhysics Conference on High Energy Physics (Brussels; 27 July - 2 August 1995) and has been communicated to the Spokesperson for each of the Collaborations (U. Timm for JADE, S.C.C. Ting for MARK-J, R. Felst for PLUTO, and G. Wolf for TASSO) and to those who presented the Collaborations' findings at the 1979 Lepton-Photon Conference (S. Orito for JADE, H.B. Newman for MARK-J, Ch. Berger for PLUTO, and G. Wolf for TASSO).

\section{EUROPEAN MOBILITY SCHEME FOR} PHYSICS STUDENTS (EMSPS)

The telnet address for the EMSPS database in Manchester giving course and general information has been changed to emspsdbs.ph.man.ac.uk.

\section{PROFESSIONAL QUALIFICATIONS}

Applications received by 1 September for admission to the European Register of Physicists (Eur Phys) will be evaluated and decided upon by the Register Commission at its first meeting in early November. The Commission will then meet approximately every six months. Applications can be submitted at any time. An application form is available from most national physical societies, from E.W.A. Lingeman (fax.: +31-20-592 21 65; ed@ nikhef.nl), and as a PostScript file (form.ps.Z) by anonymous $\mathrm{ftp}$ to www.nikhef.nl/pub/eps

\section{Edith Müller}

1918-1995

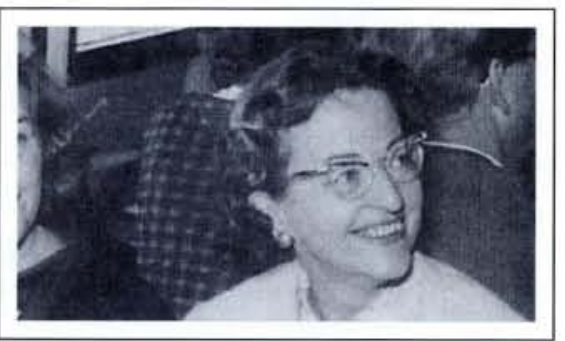

Edith Müller, a member of the EPS Executive Committee from 1978 to 1983 when she served consecutively as ViceSecretary, Treasurer and Secretary, passed away on 24 July. She was born in 1918 in Madrid where she studied before obtaining a diploma in physics and mathematics, and
Published by EPS to inform members.

Editor: P.G. Boswell, EPS, BP 69, CH-1213 Petit-Lancy 2 E-mail: peter.boswell@cern.ch Tel.: +41-22-793 11 30 - Fax: +41-22-793 13

JULY-AUGUST 1995

\section{Gilberto Bernardini 1906-1995}

It is with considerable regret that H. Schopper, the President of EPS, and R.A. Ricci, the President of the Italian Physical Society (IPS), inform members of the death on 4 August of Gilberto Bernardini a few days before his 89th birthday. Professor Bernardini, a former President of the IPS, played a leading role in founding EPS and he served as the Society's first President. An appreciation will be published in the September/October 1995 issue of Europhysics News.

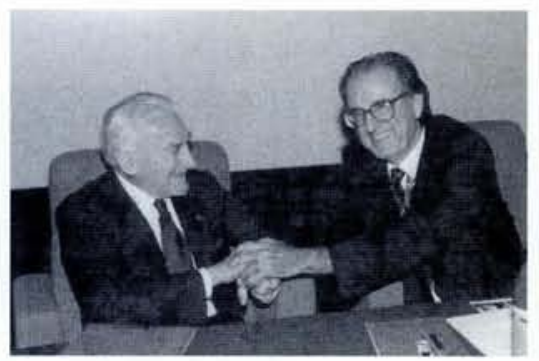

G. Bernardini, on the left, with R.A. Ricci at the special opening ceremony of the EPS-9 General Conference (Florence, 10-14 September 1993) celebrating the 25th anniversary of EPS.

subsequently a doctorate, from Zurich University. After seven years as a research assistant at the university, she worked in Cambridge, Ann Arbor (MI, USA), and Basle before becoming an Associate Professor at the University of Neuchâtel in 1962, an extraordinary Professor at the University of Geneva in 1964, and Full Professor in Geneva in 1972. A specialist in astronomical spectroscopy and in solar and stellar atmospheres, she co-authored the basic reference on elemental abundances in the Universe which was among the most quoted astronomy texts for 25 years. Professor Müller contributed greatly to the development of European solar space research, notably in experiments to measure the Sun's infrared radiation. Owing to her broad experience, she took part in many international programmes and made major contributions to the management of science. Besides being the General Secretary of the International Astronomical Union (IAU) and a member of the IAU Executive Committee, she served in various capacities with the Joint Organization for Solar Observations, the Astrophysical Centre (Canary Islands), the Royal Astronomical Society, and the Henri Chrétian Fund. Her personal qualities and her outstanding example as a teacher, organizer, and pioneering astronomer will be greatly missed.

A. Maeder, Geneva 